\title{
Non-von-Kries 3-Parameter Color Prediction
}

\author{
Brian Funt and Hao Jiang \\ School of Computing Science \\ Simon Fraser University \\ Vancouver, B.C. Canada V5A 1S6
}

\begin{abstract}
Chromatic adaptation transforms generally rely on a variant of the von Kries transformation method to account for changes in the LMS cone signals that occur when changing from one illuminant to another. Von Kries adaptation-also often referred to as the coefficient rule method or the diagonal transformation method-adjusts the 3 color channels by independent scale factors. Since there generally are only 3 known quantities available, namely the ratio of the cone signals of the two adapting illuminants, a crucial aspect of the von Kries method is that it requires only 3 parameters to be specified. A 9-parameter, 3x3 matrix transformation would be more accurate, but it is generally not possible to determine the extra parameters. This paper presents a novel method of predicting the effect a change of illumination has on the cone signals, while still relying on only 3 parameters. To begin, we create a large set of $3 \times 3$ matrices representing illuminant changes based on a sizable database of typical illuminant spectra and surface spectral reflectances. Representing these $3 \times 3$ matrices as points in a 9dimensional space, we then apply principal components analysis to find a 3-dimensional basis which best approximates the original matrix space. To model an illumination change, a $3 \times 3$ matrix is constructed using a weighted combination of the 3 basis matrices. The relative weights can be calculated based on the 3 standard cone ratios obtained from the illuminant pair. Tests show that the new method yields better results than von Kries adaptation with or without sensor sharpening.
\end{abstract}

Keywords: Chromatic adaptation, color correction, von Kries, spectral sharpening, color prediction.

\section{INTRODUCTION}

When there is a change in illumination, the cones' quantum catches change. We address the problem of predicting how they change. While the problem of predicting cone quantum catches relates to chromatic adaptation, it is not the same as chromatic adaptation. Models of chromatic adaptation, try to predict which colors will appear the same to a human subject under different illuminants. In this paper, we are concerned with predicting the LMS cone signals under a second illuminant given the LMS cone signals under a first illuminant along with the LMS cone signals of a white surface under each of the illuminants.

One common method of predicting LMS under a second illuminant is with a diagonal model of illuminant change.

Manuscript version. Final version to appear in: Proc. of SPIE Vol. 5007 Human Vision and Electronic Imaging VIII, Santa Clara, Jan. 2003.

Copyright 2003 


$$
\left[\begin{array}{c}
L_{b} \\
M_{b} \\
S_{b}
\end{array}\right]=\left[\begin{array}{ccc}
C_{L} & 0 & 0 \\
0 & C_{M} & 0 \\
0 & 0 & C_{S}
\end{array}\right]\left[\begin{array}{c}
L_{a} \\
M_{a} \\
S_{a}
\end{array}\right]
$$

This model is known as the coefficient rule or von Kries rule $e^{5,7}$. Often the coefficients $C_{i}$ are chosen as the ratio of the quantum catches obtained from a white surface under the two illuminants:

$$
C_{L}=L_{b}^{\text {white }} / L_{a}^{\text {white }}
$$

The accuracy of the diagonal model generally can be improved by including a sharpening transformation ${ }^{2}$ so that the diagonal transformation occurs in an optimal space. With sharpening transformation $T$, the diagonal model becomes:

$$
\left[\begin{array}{c}
L_{b} \\
M_{b} \\
S_{b}
\end{array}\right]=T^{-1}\left[\begin{array}{ccc}
C_{L} & 0 & 0 \\
0 & C_{M} & 0 \\
0 & 0 & C_{S}
\end{array}\right] T\left[\begin{array}{c}
L_{a} \\
M_{a} \\
S_{a}
\end{array}\right]
$$

Finlayson et. al. ${ }^{2}$ show that diagonal model with sharpening performs better than the simple diagonal model; however, its performance does not equal that of a full 9-parameter, 3x3 linear model of the form:

$$
\left[\begin{array}{c}
L_{b} \\
M_{b} \\
S_{b}
\end{array}\right]=\left[\begin{array}{lll}
C_{11} & C_{12} & C_{13} \\
C_{21} & C_{22} & C_{23} \\
C_{31} & C_{32} & C_{33}
\end{array}\right]\left[\begin{array}{c}
L_{a} \\
M_{a} \\
S_{a}
\end{array}\right]
$$

The usual reason the full $3 \times 3$ linear model is not used is that there is not enough information available to determine the 9 coefficients. In particular, if all we know about the two illuminants are the LMS values of a white surface (or something equivalent such as the average scene LMS used in the gray world method) under each illuminant then we have only 3 equations for the 9 unknowns.

The question we ask here is: Is there some other non-diagonal 3parameter model that we could use that would perform better than the sharpened diagonal model? We establish our new model by considering the 9-dimensional space of $3 \times 3$ transformations that model illuminant change and then finding the 3-dimensional subspace that best approximates it. This subspace provides a new 3-parameter, non-diagonal model of illuminant change that works better than previous models of illuminant change.

\section{COLOR PREDICTION BASED ON PRINCIPLE COMPONENT ANALYSIS}

Manuscript version. Final version to appear in: Proc. of SPIE Vol. 5007 Human Vision and Electronic Imaging VIII, Santa Clara, Jan. 2003. 
In the color prediction problem, we are going to estimate the color of a surface under different illuminations. In this process, we assume matte surfaces and ignore changes due to shading since by a change of illumination we mean only a change in the spectral composition of the illumination, not a change in the illuminant's position. The quantum catch at single point on the surface as determined by the incoming spectrum of the illumination and the surface reflectance is then

$$
h_{i}=\int_{\lambda} E(\lambda) S_{i}(\lambda) R(\lambda) d \lambda,
$$

where $E(\lambda)$ is the spectrum of the illumination, $R(\lambda)$ is the percent surface spectral reflectance function, $S_{i}(\lambda), i=1,2,3$ are the sensor or cone sensitivities.

Assume that the lighting spectrum can be expanded based on an arbitrary set of orthogonal bases as,

$$
E(\lambda)=e_{0} f_{0}(\lambda)+e_{1} f_{1}(\lambda)+\ldots+e_{n} f_{n}(\lambda) .
$$

Then the quantum catches on three channels corresponding to the sensors are,

$$
h_{i}=\sum_{j=0}^{n} e_{j} \int_{\lambda} S_{i}(\lambda) f_{j}(\lambda) R(\lambda) d \lambda .
$$

Rewriting in matrix form results in

$$
h=A e,
$$

where $A$ is a $3 \times n$ matrix which is independent of the illumination. If two illuminant spectra have a linear relationship, say $e_{b}=M e_{a}$, where $M$ is an n-by-n transformation matrix then we have,

$$
h_{b}=A M e_{a} \text {, }
$$

For three-dimensional illuminants, we can swap the position of $A$ and $M$ to yield

$h_{b}=M A e_{a}=M h_{a}$.

Since $M$ is independent of the surface reflectance, this relationship applies to all surfaces under the two illuminants so $M$ models illumination change.

If we write the elements of the $3-b y-3 M$ out as a vector, the space of such matrices is 9 dimensional. However, what is the underlying dimensionality of matrices $M$ ? Might the 9-dimensional space be embedded in a lower dimensional space of dimension as low as three? Since we know that color prediction based on diagonal matrix works quite well, it seems reasonable to expect the dimensionality of $M$ to be much lower than 9. Instead of forcing the 3 parameters to be those of a diagonal matrix, we use principal component analysis to estimate the real dimensionality of the transformation matrix $M$ and extract the optimum 3-parameter linear color prediction method.

To determine the dimensionality of the space of illumination transformation matrices, $M$, we first constructed a large set of

Manuscript version. Final version to appear in: Proc. of SPIE Vol. 5007 Human Vision and Electronic Imaging VIII, Santa Clara, Jan. 2003. 
corresponding quantum catches under various illuminant pairs. These pairs of illuminants were chosen from a set of 140 different illuminants in the Simon Fraser University database ${ }^{1}$. All the illuminants were normalized to unit energy. For each illuminant pair, the corresponding quantum catches for 1995 surface reflectances from the Kodak reflectance database ${ }^{1,6}$ were calculated. The best, in the least-squares sense, 3-by-3 illumination transformation matrix, $M$, mapping one set to the other is then calculated. For $n$ illuminant pairs, we have $n$ new such matrices $M$.

We applied principal components analysis to the set of matrices $M$. To do so, we first write each $M$ as a vector $m$ by scanning the matrix row by row. Arranging all such 9-element vectors as rows in a matrix results in an n-by-9 matrix $S$. Principal components analysis of $S$ produces basis vectors $v_{i}, i=1, \ldots, 9$. Let the mean $m$ vector be $m_{0}$. These vectors can be reshaped back into corresponding $3 \times 3$ matrices $V_{i}$ and $M_{0}$.

An illumination transformation matrix $M$ can then be represented as

$$
M=\sum_{i=1}^{9} c_{i} V_{i}+M_{0}
$$

where $c_{i}=\left(m-m_{0}\right) \cdot v_{i}$.

We can also approximate $M$ by truncating the summation and using fewer than 9 basis matrices $V_{i}$. Figure 1 shows the residual error in approximating all matrices $M$ as the number of basis matrices is increased.

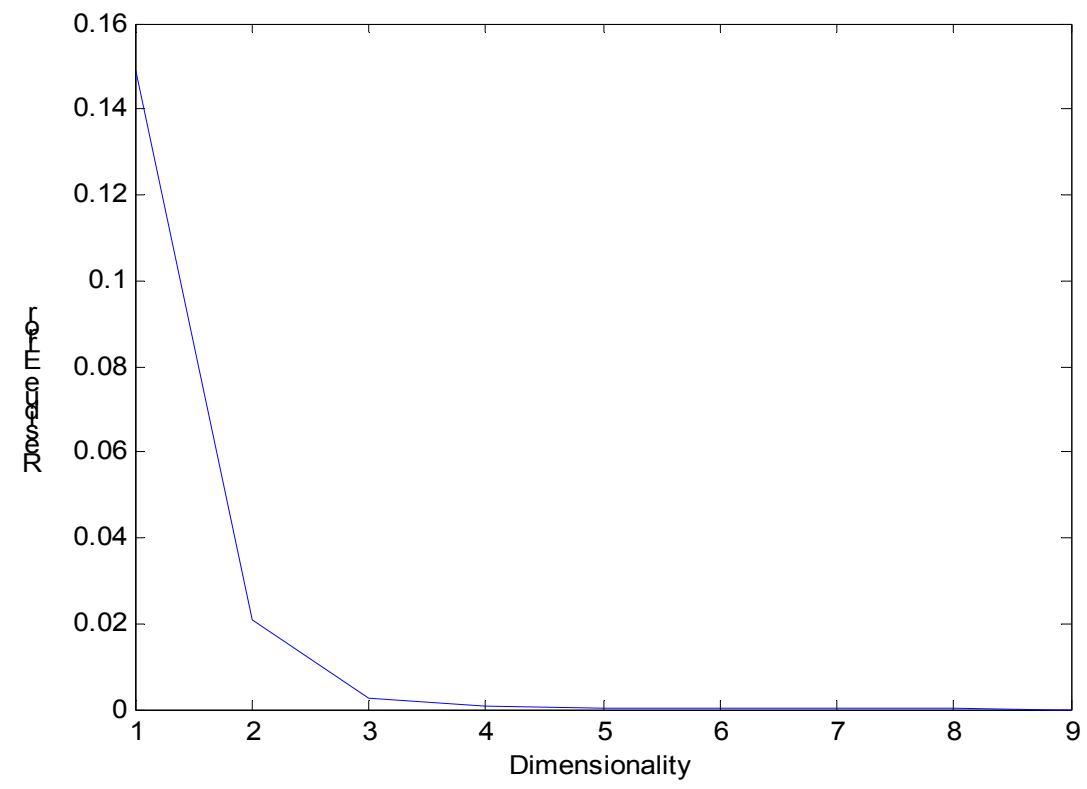

Figure 1. Residual error as a function of dimension

Manuscript version. Final version to appear in: Proc. of SPIE Vol. 5007 Human Vision and Electronic Imaging VIII, Santa Clara, Jan. 2003. 
More than 99\% of the total energy is concentrated in the first 3 degrees. As expected, a 3-dimensional model of illumination change is very good. The remaining issue is how to use this model for color prediction.

Based on principal component analysis, we have the first three bases matrices denoted as $V_{1}, V_{2}$ and $V_{3} . M$ is approximated as $M=c_{1} V_{1}+c_{2} V_{2}+c_{3} V_{3}+M_{0}$. Then given the LMS 3-vectors, $l_{a}$ and $l_{b}$, of white under the two illuminants, the coefficients $c_{i}$ required to predict colors under illumination b from colors under illumination a can be determined as follows. Since $l_{b}=l_{a} M$, we have

$$
\begin{aligned}
l_{b}-l_{a} M_{0}= & c_{1} l_{a} V_{1}+c_{2} l_{a} V_{2}+c_{3} l_{a} V_{3} \\
= & c_{1}\left[l_{a} V_{1}(1), l_{a} V_{1}(2), l_{a} V_{1}(3)\right]+c_{2}\left[l_{a} V_{2}(1), l_{a} V_{2}(2), l_{a} V_{2}(3)\right]+ \\
& c_{3}\left[l_{a} V_{3}(1), l_{a} V_{3}(2), l_{a} V_{3}(3)\right] \\
= & {\left[c_{1}, c_{2}, c_{3}\right] Q }
\end{aligned}
$$

with

$$
Q=\left[\begin{array}{lll}
l_{a} V_{1}(1) & l_{a} V_{1}(2) & l_{a} V_{1}(3) \\
l_{a} V_{2}(1) & l_{a} V_{2}(2) & l_{a} V_{2}(3) \\
l_{a} V_{3}(1) & l_{a} V_{3}(2) & l_{a} V_{3}(3)
\end{array}\right]
$$

The notation $V_{i}(j)$ denotes the $j^{\text {th }}$ column of matrix $V_{i}$.

Letting $c=\left[c_{1}, c_{2}, c_{3}\right]$, we have,

$$
c=\left(l_{b}-l_{a} M_{0}\right) Q^{-1}
$$

Diagonal matrix color prediction can be viewed as a special case in which the base matrices are simply

$$
\left[\begin{array}{lll}
1 & 0 & 0 \\
0 & 0 & 0 \\
0 & 0 & 0
\end{array}\right],\left[\begin{array}{lll}
0 & 0 & 0 \\
0 & 1 & 0 \\
0 & 0 & 0
\end{array}\right],\left[\begin{array}{lll}
0 & 0 & 0 \\
0 & 0 & 0 \\
0 & 0 & 1
\end{array}\right]
$$

\section{EXPERIMENTAL RESULTS}

Experiments were carried out to verify the performance of the new nonvon-Kries, PCA-based color prediction scheme. We compare the new method to diagonal-matrix color prediction and to sharpened diagonal prediction. The data in the experiment is generated based on the same database of 140 different illuminants and 1995 different surfaces described above.

In the first experiment, we use all the data in the database for both training (i.e., deriving the optimal basis matrices) and for testing. Table 1 illustrates the comparative results based on the absolute L1

Manuscript version. Final version to appear in: Proc. of SPIE Vol. 5007 Human Vision and Electronic Imaging VIII, Santa Clara, Jan. 2003. 
norm error. The row labeled "Full Linear" is based on color prediction with a full 3-by-3 matrix fit to the training data. It represents the best possible linear prediction and acts as a benchmark. The Sharpened Diagonal result is based on randomly selecting ten illumination pairs for calculating the sharpening matrix and using the one that results in the least error. As can be seen from the table, the new non-von-Kries PCA-based method gives the best result across all three channels.

\begin{tabular}{llll}
\hline \hline & $\mathrm{L}$ & $\mathrm{M}$ & $\mathrm{S}$ \\
\hline Diagonal & 2.3611 & 1.1931 & 0.0048 \\
Sharpened Diagonal & 1.1449 & 0.7478 & 0.0070 \\
New non-von-Kries & 0.9525 & 0.5920 & 0.0049 \\
Full Linear & 0.6079 & 0.3275 & 0.0030 \\
\hline \hline
\end{tabular}

Table 1 Comparison of the average absolute error for the case in which all the data in the database is used for both training and testing.

Table 2 shows the average relative error where again the new method is best and Table 3 gives the error in CIE L*a* $\mathrm{b}^{*} \Delta \mathrm{E}$.

\begin{tabular}{llll}
\hline \hline & $\mathrm{L}$ & $\mathrm{M}$ & $\mathrm{S}$ \\
\hline Diagonal method & 0.0455 & 0.0508 & 0.0151 \\
Sharpening method & 0.0243 & 0.0318 & 0.0299 \\
New non-von-Kries & 0.0200 & 0.0274 & 0.0181 \\
Full Linear & 0.0132 & 0.0175 & 0.0142 \\
\hline \hline
\end{tabular}

Table 2 Relative error comparison results with all the data in the database for training and testing.

\begin{tabular}{ll}
\hline \hline Diagonal method & 0.9335 \\
Sharpening method & 0.3609 \\
New non-von-Kries & 0.2085 \\
\hline \hline
\end{tabular}

Table 3 Comparison of results using the average CIE $\mathrm{L}^{*} \mathrm{a}^{*} \mathrm{~b}^{*} \Delta \mathrm{E}$ error for the case in which all the data in the database is used for both training and testing.

In a second experiment, we randomly selected 100 illuminants for training and the rest of the data, which include 40 other illuminants, for testing. All 1995 surface reflectances were used for both training and testing. The results presented in Tables 4, 5 and 6 show that new non-von-Kries method still performs the best whether measured in terms of absolute error or CIE L $\mathrm{L}^{*} \mathrm{~b}^{*} \Delta \mathrm{E}$ error.

For the sharpening method, 50 randomly selected illumination pairs were used when calculating the sharpening matrix. Since sharpening is derived from a single illuminant pair, when applied to color prediction on different illuminant pairs the results vary. Table 4 shows how the error varies with the training pair.

Manuscript version. Final version to appear in: Proc. of SPIE Vol. 5007 Human Vision and Electronic Imaging VIII, Santa Clara, Jan. 2003. 


\begin{tabular}{lllll}
\hline \hline & & L & M & S \\
\hline Mean of Mean Absolute Prediction Error & 1.7658 & 1.3073 & 0.0174 \\
Std Dev of Mean Prediction Error & 2.7687 & 1.7376 & 0.0379 \\
Maximum of Mean Prediction Error & 19.3155 & 12.2909 & 0.2718 \\
Minimum of Mean Prediction Error & 0.9147 & 0.6586 & 0.0059 \\
\hline \hline
\end{tabular}

Table 4 The variation in the absolute color prediction error for different sharpening matrices. The sharpening matrices were calculated using 50 different randomly selected illuminant pair. For each pair the mean color prediction error is computed.

\begin{tabular}{|c|c|c|c|}
\hline & L & $\mathrm{M}$ & $\mathrm{S}$ \\
\hline Diagonal method & 1.9501 & 1.0650 & 0.0059 \\
\hline Sharpening method (Best L1) & 0.9147 & 0.6586 & 0.0059 \\
\hline New non-von-Kries & 0.8839 & 0.5813 & 0.0053 \\
\hline Eull Linear & 0.7103 & 0.4128 & 0.0041 \\
\hline
\end{tabular}

Table 5 Absolute error comparison results with 100 illuminants for training and 40 illuminants for testing. The sharpening method is given the benefit of the doubt by choosing the sharpening matrix that yields the best results in Table 4.

\begin{tabular}{ll}
\hline \hline Diagonal method & 0.6836 \\
Sharpening method (best L1) & 0.2444 \\
New non-von-Kries & 0.2050 \\
Full Linear & 0.1327 \\
\hline \hline
\end{tabular}

Table 6 CIE L* ${ }^{*} b^{*} \Delta E$ error with 100 illuminants for training and 40 for testing.

In the third experiment, we randomly selected 100 illuminants and 1000 reflectances for training. The testing data included the remaining 40 illuminants and 995 surfaces from the database. As before, the new nonvon-Kries method has the least absolute, relative and CIE $\mathrm{L}^{*} \mathrm{a}^{*} \mathrm{~b}^{*} \Delta \mathrm{E}$ error. Table 7 shows the CIE L*a $\mathrm{b}^{*} \Delta \mathrm{E}$ case.

\begin{tabular}{ll}
\hline \hline Diagonal method & 0.4752 \\
Sharpening method (best L1) & 0.1707 \\
New non-von-Kries & 0.1410 \\
Full Linear & 0.0790 \\
\hline
\end{tabular}

Table 7. CIE L ${ }^{*} a^{*} b^{*} \Delta E$ error comparison for the case of 100 illuminants and 1000 reflectances used in training with testing on 40 illuminants and 995 reflectances.

\section{CONCLUSION}

Manuscript version. Final version to appear in: Proc. of SPIE Vol. 5007 Human Vision and Electronic Imaging VIII, Santa Clara, Jan. 2003. 


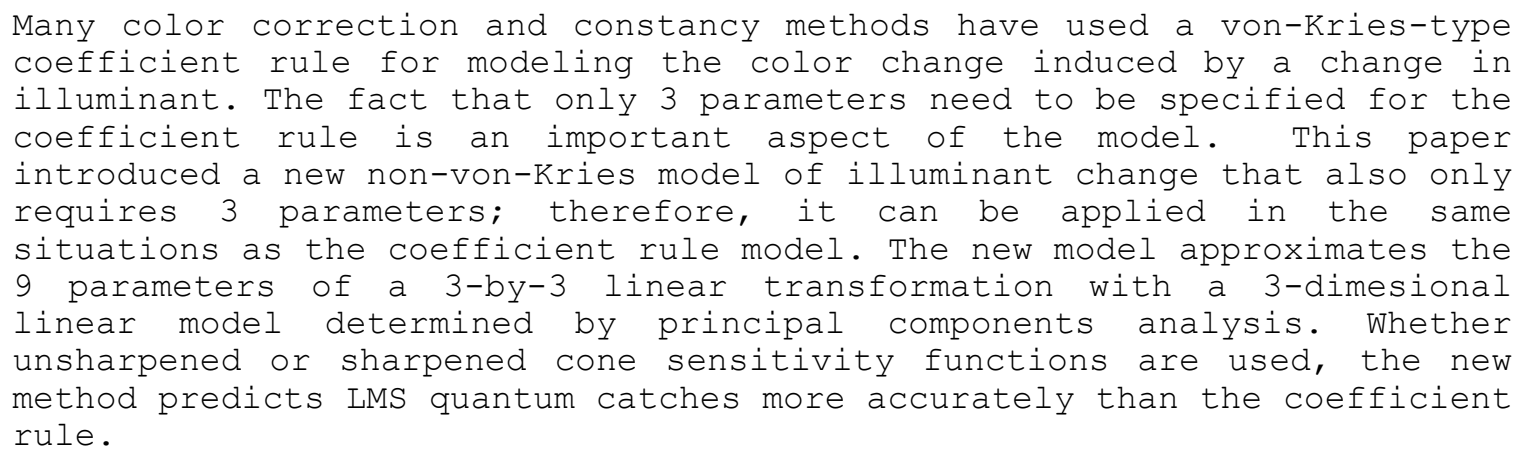

\section{ACKNOWLEDGEMENT}

Funding for this project was provided by the Natural sciences and

Engineering Research Council of Canada.

\section{REFERENCES}

1. K. Barnard, L. Martin, B. Funt and A. Coath, "A Data Set for Colour Research," Color Research and Application, Vol. 27, No. 3, pp. 147-151, 2002,

http://www.cs.sfu.ca/ colour/data/colour_constancy_synthetic_test_data/index.html

2. G.D. Finlayson, M. Drew and B. Funt, "Spectral Sharpening: Sensor Transformations for Improved Color Constancy," Journal of Optical Society of America A, Vol. 11, No. 5, pp. 1553-1563, 1994

3. K. M. Lam, "Metamerism and colour constancy," Ph.D. Thesis, University of Bradford, 1985.

4. M.R. Luo and R.W.G. Hunt, "A chromatic adaptation transform and a colour inconstancy index," Color Res. Appl., 23, pp. 154-158, 1998.

5. J. von Kries, "Chromatic Adaptation" originally published in Festschrift der Albrecht-LudwigsUniversitat (1902), In MacAdam, D.L. Ed. Sources of Color Vision, MIT Press, Cambridge, 1970

6. M.J., Vrhel, R. Gershon, R. Iwan, L. S. "Measurement and analysis of object reflectance spectra," Color Res. Appl., 19(1) pp. 4-9, 1994.

7. J.A. Worthey and M.H. Brill, "Heuristic Analysis of von Kries color constancy," J. Opt. Soc. Am. A 3, 1708-1712 (1986).

Manuscript version. Final version to appear in: Proc. of SPIE Vol. 5007 Human Vision and Electronic Imaging VIII, Santa Clara, Jan. 2003. 